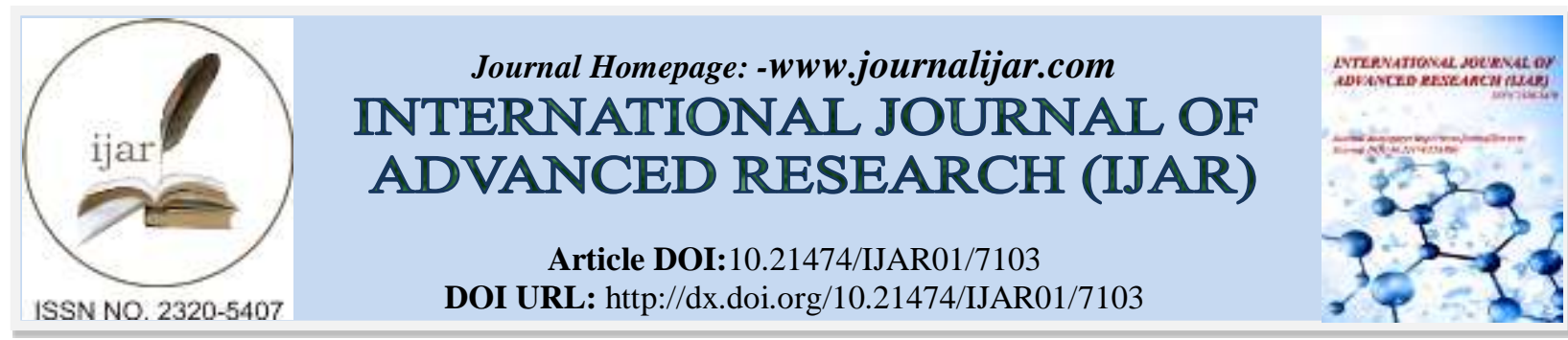

RESEARCH ARTICLE

\title{
CANCER DU SEIN CHEZ LA FEMME ÂGÉE : EXPERIENCE DU SERVICE D'ONCO- RADIOTHÉRAPIE CHU DE MARRAKECH BREAST CANCER IN ELDERLY WOMEN: EXPERIENCE OF THE SERVICE OF ONCO-RADIOTHERAPY UNIVERSITY HOSPITAL OF MARRAKECH.
}

N. Bouzid, K.diakité, A. Elomrani and M. Khouchani.

Service d' Onco- Radiothérapie CHU Mohammed VI, Marrakech.

\section{Manuscript Info}

Manuscript History

Received: 15 March 2018

Final Accepted: 17 April 2018

Published: May 2018

Keywords:-

breast cancer -old woman-managementprognosis

\begin{abstract}
Our objective was to analyze the epidemiological, clinical, therapeutic and prognostic of breast cancer in older women. We performed a retrospective study of 35 cases of breast cancer among older women treated and followed in the oncology radiotherapy department of the University Hospital Mohammed VI Marrakech, in over a period of 5 years from 2012 to 2016.Among 603 women who had breast cancer during the study period, 35 were aged 65 and over, or 5,8\%. The average age of these patients was 72-year-old. The mean time for diagnosis was 15 months. A breast nodule was the main sign in $91,42 \%$ of cases, associated with inflammatory signs in five cases. The majority of tumors were classified T3 $(51,43 \%)$ forms classified T4 represented $25,42 \%$ of the tumors. Ipsilateral axillary lymph nodes found in 14 patients or $40 \%$ of cases. The disease was metastatic at diagnosis in 5 cases. Infiltrative ductal carcinoma represented $88,57 \%$. It was in $74,28 \%$ of cases of grade II Scarff-Bloom and Richardson (SBR), and $25,72 \%$ grade III. The lymph node was positive in 22 patients $(62,85 \%)$. The expression of HR was noted in $74,28 \%$ of cases. HER2 was over expressed in $14,28 \%$ of cases. Surgical treatment was performed after neo adjuvant chemotherapy in 7 cases of locally advanced cancers, three patients underwent conservative treatment, while 31 patients underwent a mastectomy with lymphnode dissection, associated with radiation therapy in $77,14 \%$ of cases and a profit analgesic radiotherapy in 2 patients. Adjuvant Chemotherapy was prescribed for 20 patients. $74,28 \%$ of the patients received hormonal therapy. Trastuzumab was given in $11,42 \%$ of cases. The evolution was marked by a complete remission after a decline of a year in 20 cases, 5 patients had progressive disease after a mean of ten months. Conclusion: The age factor is not to be alone a criterion for treatment decisions, understanding the multiple facets of the physical and psychological health of the patient and the medical, social and family should be taken into account.
\end{abstract}

Copy Right, IJAR, 2018, All rights reserved. 


\section{Introduction:-}

Le cancer du sein est le cancer le plus fréquent chez la femme. Il représente la première cause de mortalité et de morbidité par cancer chez la femme dans toutes les tranches d'âge. Du fait de l'augmentation de l'espérance de vie de la femme marocaine et, en parallèle, l'incidence croissante des cancers avec l'âge, les femmes âgées représentent une importante proportion des patientes atteintes de cancer du sein, ce qui fait de cette pathologie un problème majeur de santé publique.

Le but de ce travail est de préciser, à travers l'étude d'une série de 35 cas et une revue de la littérature, les caractéristiques épidémiologiques, cliniques, anatomopathologiques, thérapeutiques et pronostiques du cancer du sein chez cette catégorie d'âge, d'évaluer les résultats des différents traitements et de ressortir les facteurs de mauvais pronostic et les moyens pouvant les améliorer.

\section{Matériels et Méthodes:-}

Cette étude rétrospective porte sur une série de 35 cas de cancer du sein chez les femmes âgées colligés dans notre service entre 2012 à 2016. Pour chaque patiente, nous avons examiné les antécédents personnels et familiaux, l'histoire clinique, les données anatomopathologiques, thérapeutiques et évolutives. Les tumeurs ont été classées selon la classification TNM de l'Union internationale de lutte contre le cancer (UICC) 2010. L'analyse informatique des différentes variables a été réalisée par le logiciel de biostatistique SPSS dans sa version 10.0.

\section{Résultats:-}

Epidémiologie:

Parmi 603 femmes ayant eu un cancer du sein durant la période de l'étude, 35 étaient âgées de 65 ans et plus, soit 5,8\%. La moyenne d'âge de nos patientes est de 72 ans, avec des extrêmes allant de 65 ans à 80 ans, $68,25 \%$ avaient plus de 70 ans. L'origine géographique a été urbaine dans $86,66 \%$. Concernant le terrain, l'HTA a été notée chez $28,75 \%$ des patientes et le diabète chez 17,44\%. Aucune de nos malades n'avait d'antécédent personnel de cancer gynécologique. Deux patientes avaient un antécédent de maladie fibro-kystique du sein, et deux patientes avaient un antécédent familial au premier degré de néoplasie du sein. L'index de Karnofsky était supérieur ou égal à $80 \%$ chez $37,14 \%$ malades. Pour ce qui est statut hormonal, L’âge moyen de la ménarche dans notre série était de 13 ans avec des extrêmes allant de 10 à 16 ans. Plus de 34,28\% des malades ont eu leurs premières règles avant l'âge de 12 ans. L'âge moyen de ménopause dans notre série était de 52 ans. 40\% des patientes étaient ménopausées après l'âge de 55 ans. La majorité de nos malades étaient multiparts et la nulliparité a été notée dans 8,57\% des cas. Deux patientes avaient eu leur première grossesse après l'âge de 30 ans. 11,42\% des patientes n'avaient pas eu recours à l'allaitement maternel. Aucune de nos malades n'a suivi de traitement hormonal substitutif.

\section{Clinique:}

Délai moyen de consultation des malades était de 15 mois, avec des extrêmes allant de 4 à 24 mois. Le motif de consultation a été la découverte d'un nodule du sein à l'autopalpation dans $91,42 \%$ des cas (32 cas), il a été associé à une mastodynie dans 4 cas et à un écoulement mamelonnaire dans deux cas. Cinq patientes avaient au moment du diagnostic des signes inflammatoires. La taille tumorale moyenne a été de 5,6 cm avec des extrêmes allant de 1 à 12 $\mathrm{cm}$. La tumeur était de volume supérieur à $5 \mathrm{~cm}$ dans 18 cas $(51,43 \%)$. Des adénopathies axillaires homolatérales retrouvés chez 14 patientes et elles étaient mobiles chez 11 patientes $(31,42 \%)$. Deux malades avaient des adénopathies sus-claviculaires.

\section{Paraclinique:}

La mammographie associée ou non à une échographie mammaire a été réalisé chez toutes nos patientes, Chez 33 patientes, ces bilans radiologiques ont pu mettre en évidence une opacité suspecte isolée. Ils ont révélé un nodule associé à des microcalcifications dans deux cas.

\section{Anatomopathologie:}

Le type histologique le plus fréquent était le carcinome canalaire infiltrant $(88,57 \%$ des cas). Le grade histopronostique (SBR) le plus observé dans notre série a été le SBR II qui a été noté chez 74,28\% des malades. L'envahissement ganglionnaire était positif chez 22 patientes $(62,85 \%)$. Les récepteurs hormonaux étaient positifs chez 74,28\% de nos patientes et l'hercept test était négatif dans 85,72\% (Tableau I). 


\begin{tabular}{|l|l|l|}
\hline Carcinome canalaire infiltrant & 30 & 86,68 \\
Carcinome lobulaire infiltrant & 2 & 6,66 \\
Carcinome mucineux & 1 & 3,34 \\
Carcinome indifférencié & 1 & 3,33 \\
\hline Grade SBR & & \\
\hline Grade I & 0 & 0 \\
Grade II & 26 & 74,28 \\
Grade III & 9 & 25,72 \\
\hline Envahissement ganglionnaire & & \\
\hline N+ & 22 & 62,85 \\
N- & 13 & 37,15 \\
\hline Emboles vasculaires & 6 & 17,14 \\
\hline Récepteurs hormonaux & & \\
\hline RE et RP positifs & 14 & 40 \\
RE positifs et RP négatifs & 7 & 20 \\
RE négatifs et RP positifs & 5 & 14,28 \\
RE et RP négatifs & 9 & 25,71 \\
\hline Hercept test & & \\
\hline Her2 négatif & 30 & 85,2 \\
Her2 positif & 5 & 14,28 \\
\hline
\end{tabular}

Tableau I :- Les caractéristiques anatomopathologies des patientes

\section{Bilan D'extension :-}

Toutes nos patientes ont fait un bilan d'extension minimal consistant en une radiographie thoracique et une échographie abdomino-pelvienne. Les métastases à distance ont été d'emblée diagnostiquées chez 5 patientes $(14,28 \%)$ dont 2 localisations hépatiques et une pulmonaire toutes infirmées par la TDM. La scintigraphie osseuse a été réalisée pour 14 patientes et a objectivé des lésions secondaires pour 2 cas.

Selon la classification TNM de l'UICC 2010 ; la majorité des tumeurs $(51,43 \%)$ ont été classées T3, Les formes classées T4 ont représenté $25,72 \%$ des tumeurs dont 4 cas classés T4b et 5 cas T4d. $40 \%$ des patientes avaient des adénopathies axillaires homolatérales. Deux malades avaient des adénopathies sus-claviculaires. (Tableau II)

\begin{tabular}{|l|c|c|}
\hline TNM & Nombre de cas & Pourcentage(\%) \\
\hline T & & \\
T1 & 1 & 2,85 \\
T2 & 7 & 20 \\
T3 & 18 & 51,43 \\
T4 & 9 & 25,72 \\
\hline N & & \\
N0 & 19 & 54,28 \\
N1 & 11 & 31,43 \\
N2 & 3 & 8,57 \\
N3 & 2 & 5,71 \\
\hline M & 30 & 85,72 \\
M0 & 5 & 14,28 \\
\hline
\end{tabular}

Tableau II:- Répartition des malades selon le TNM

\section{Traitement:}

Toutes nos patientes ont eu un geste chirurgical. Parmi les patientes opérées, sept avaient eu une chimiothérapie néoadjuvante. Cette chirurgie a été radicale (La mastectomie avec curage axillaire) chez 31 patientes. 3 malades ont bénéficié d'une chirurgie conservatrice. Une mastectomie de propreté a été réalisée chez une patiente.

L'irradiation complémentaire a été réalisé chez $77,14 \%$ des patientes, une radiothérapie palliative a été indiqué chez 2 patientes, une patiente a bénéficié d'un protocole hypofractionné. Aucune complication grave n'a été notée. 
La chimiothérapie adjuvante a été prescrite chez 20 malades (57,14\%), et l'indication était purement palliative chez deux patientes.

L'hormonothérapie adjuvante a été indiquée chez $65,71 \%$ des cas pendant cinq ans. Les anti-aromatases ont été utilisés chez 18 malades et 5 patientes étaient sous tamoxifène. 3 patientes ont été mise sous hormonothérapie palliative.

Parmi les 5 malades qui avaient une surexpression du récepteur HER2, quatre ont Bénéficié d'une thérapie ciblée à base du trastuzumab.

\section{Evolution:-}

L'évolution après le traitement de première intention a été marquée par une rémission complète après un recul d'une année dans 20 cas et cinq patientes avaient une maladie en progression après un recul moyen de dix mois. Pour le reste des patientes ; 4 patientes sont décédées et 6 patientes ont été perdues de vue.

\section{Discussion:-}

Le cancer du sein est un problème de santé majeur dans le monde. L'âge est le facteur de risque le plus connu du cancer en général et du cancer du sein en particulier. L'âge médian de survenue des cancers du sein aux États-Unis est désormais de 61 ans d'après les dernières données des registres de Surveillance Epidemiology and End Results (SEER), ce qui représente environ 15\% de la population américaine. En Europe, pour les femmes âgées de 50 à 54 ans, l'incidence de cancer du sein est de 210 par 100.000 femmes, montant à plus de 300 par 100.000 femmes à l'âge de 70 ans et à plus de 430 par 100.000 femmes pour celles âgés de plus de 80 ans [1]. Au Maroc, selon le rapport du Haut-Commissariat au Plan de 2007, l'espérance de vie à la naissance des femmes est de 73,67 ans. En 2004, la proportion des personnes âgées de 60 ans et plus était de 8,1\% [2]. Dans notre étude 5,8\% des patientes ayant un cancer du sein étaient âgées de 65 ans et plus. L'âge moyen retrouvé dans la littérature est de 70 à 73 ans chez les patientes âgées de plus de 65 ans [3]. Dans notre étude il était de 72 ans rejoignant ainsi les données de la littérature.

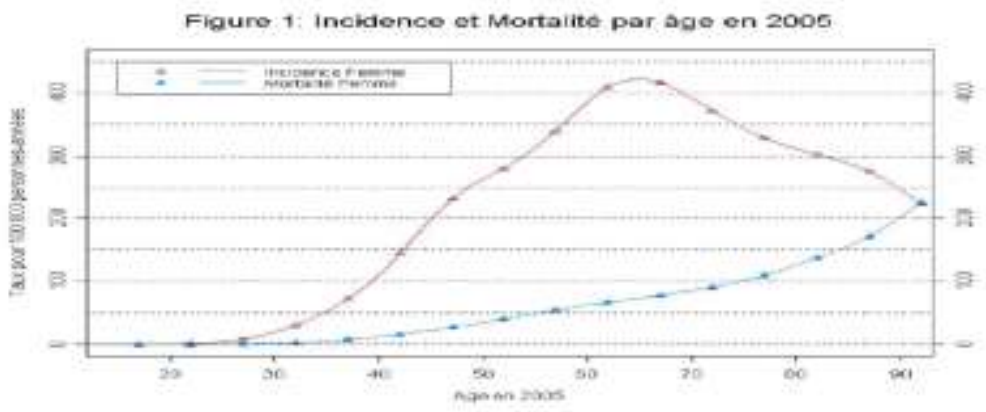

Les comorbidités représentent le facteur majeur limitant la possibilité de se prononcer par rapport au pronostic du cancer du sein chez les personnes âgées. En effet, quand leur nombre augmente, le nombre de décès dus à des causes intercurrentes dépasse le nombre de décès dus au cancer en lui-même [4]. Dans une étude menée par Gironès en Espagne [5] sur 91 patientes âgées de plus de 70 ans atteintes de cancer du sein, la pathologie cardio-vasculaire et le diabète représentaient les comorbidités les plus souvent retrouvées.

Pour le statut hormonal, Plusieurs études incriminent l'âge des premières règles comme facteur de risque, James [6] a trouvé qu'après 65 ans, une ménarche précoce inférieure à 12 ans n'augmente pas le risque de cancer du sein alors qu'une puberté tardive confère une protection. Un âge avancé à la ménopause ressort souvent aussi comme facteur de risque de développer un cancer du sein. La ménopause précoce est protectrice surtout chez les femmes de plus de 70 ans [7]. Concernant l'âge des premières naissances; les données de la littérature sont concordantes pour accorder à l'âge lors de la première grossesse et au nombre d'enfants un rôle important dans la genèse du cancer du sein. Alors que le risque de cancer du sein augmente lors d'une première grossesse tardive, ce risque est surtout présent pour les cancers survenant chez les femmes âgées, de même, la nulliparité a été retenue dans la littérature comme un facteur de risque de cancer du sein même chez les femmes âgées [9]. Dans notre série, seulement 8,57\% de nos malades sont nullipares et seulement deux patientes ont eu leur grossesse après l'âge de 30 ans. 
L'antécédent familial de cancer du sein ne joue apparemment pas ou peu pour les femmes âgées puisque les cancers du sein familiaux apparaissent généralement avant 65 ans. Selon Bignon, la probabilité de trouver un cancer du sein lié à une mutation de type BRCA-1 ou BRCA-2 n'est que de 3\% chez les femmes de plus de 70 ans et de $1 \%$ chez les femmes de plus de 80 ans.

Le délai moyen de consultation montre que les femmes âgées consultent généralement dans un délai plus long que les femmes jeunes. En effet, en France environ $20 \%$ des patientes âgées attendent près d'un an avant de consulter pour des symptômes identifiés [10]. Ce retard diagnostique peut être expliqué par : les comorbidités (le fait qu'un symptôme nouveau puisse être interprété comme une conséquence du vieillissement ou attribué à une pathologie préexistante), par la difficulté d'accès aux soins, par des problèmes sociaux ou financiers ou par les problèmes psychologiques de la femme âgée. Ce retard de diagnostic pourrait expliquer le stade avancé au moment du diagnostic.

L'autopalpation d'un nodule du sein a été le motif de révélation le plus fréquent. Martin [11] a trouvé que les patientes âgées de 70 à 74 ans ont découvert elles-mêmes un nodule dans $51 \%$ des cas contre $30 \%$ pour les femmes âgées de 75 à 85 ans. Pour Hieken [12], le nodule du sein était révélateur du diagnostic chez 58\% de ses malades âgées de plus de 65 ans. Dans notre série le nodule du sein a été un motif de consultation presque constant, et ce chez 32 patientes.

Suivant les séries, la taille des tumeurs varie. Pour Truong (Canada) [13] et Chapgar (USA) [14], plus de la moitié des malades présentent une tumeur mesurant moins de $2 \mathrm{~cm}$. Pour Lee (Canada), la majorité des patientes présentaient une tumeur évoluée, mesurant plus de $5 \mathrm{~cm}$ [15]. Les données de notre série rejoignent ces derniers résultats. $51,43 \%$ de nos malades se présentent à un stade très évolué où la tumeur dépasse $5 \mathrm{~cm}$, ceci témoignant du retard diagnostique précédemment constaté.

Les métastases viscérales s'avèrent plus fréquentes chez la femme âgée [16] ce qui est concordant aux résultats de notre série $(14,28 \%$ des cas).

Sur le plan histologique, le CCI est le type histologique le plus fréquent, il est noté dans la plupart des séries [17]. Chez la femme âgée, tous les auteurs s'accordent à dire qu'il y a moins de grade III par rapport à la femme jeune. David [18] a trouvé un grade SBR élevé chez les femmes jeunes non ménopausées dans 67,1\%, chez les femmes âgées non ménopausées dans $52 \%$ et chez les femmes âgées ménopausées dans 39,9\%. Nous avons trouvé chez $74,28 \%$ cas un grade II contre $25,72 \%$ de grade III.

Concernant le taux d'envahissement ganglionnaire, les résultats des différentes publications divergentes Fisher et Jaquemier [23-24] ont observé un envahissement ganglionnaire moins fréquent dans la population des femmes âgées de plus de 70 ans. Pour d'autres comme Daidone [25] et Gennari [26], il augmenterait après l'âge de 65 ans. Le taux d'envahissement ganglionnaire chez nos patientes était de $62,85 \%$.

Sur le plan moléculaire La majorité des auteurs trouvent chez la femme âgée des taux des récepteurs hormonaux positifs plus fréquents [19]. En effet, la fréquence des tumeurs n'exprimant pas de récepteurs hormonaux est inférieure à $20 \%$ après l'âge de 70 ans, comparée à $50 \%$ chez les patientes de plus de 20 ans [20] (fig. 1). Dans notre étude Les récepteurs hormonaux étaient positifs chez $74,28 \%$ de nos patientes. 


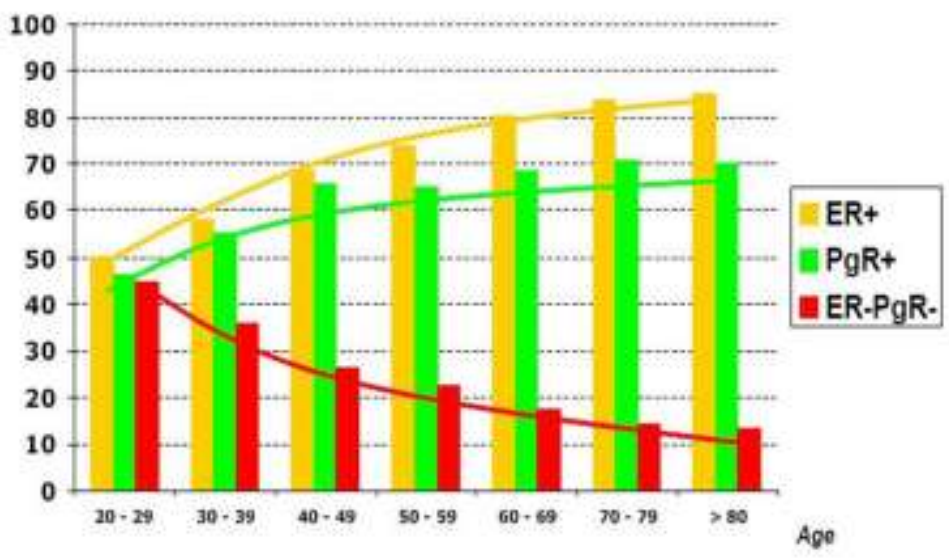

Figure 1:- les taux de récepteurs hormonaux selon l'âge [20]

L'expression de l'HER2 est faible chez les femmes âgées, comme le démontre Durbecq [21] dans sa série où 7\% des malades de plus de 70 ans expriment l'HER 2 contre $15 \%$ des malades de moins de 50 ans. Dans notre étude l'hercept test était positif dans $14,28 \%$.

Le taux d'expression du Ki 67 par les tumeurs chez les femmes âgées est plus faible comparé aux femmes plus jeunes. Ceci est confirmé par l'étude de Ma [22] en Chine, puisqu'il est positif chez $14 \%$ des malades âgées de 70 à 74 ans et chez 8\% des malades de plus de 75 ans, contre $60 \%$ des malades âgées de 60 à 64 ans.

La prise en charge du cancer du sein chez la femme âgée devrait être adaptée à ses problèmes spécifiques y compris ceux liés à l'espérance de vie, à l'âge physiologique et aux conditions physiques. Il n'existe pas de stratégie thérapeutique propre aux femmes âgées. Les mêmes conduites sont appliquées, tout en tenant compte des tares éventuelles [27].

Les contre-indications absolues à la chirurgie chez la femme âgée sont exceptionnelles et résultent de co-morbidités lourdes et multiples. Le taux du traitement conservateur diminue avec l'âge à stade égal de la maladie [28]. Solin [29], n'a pas trouvé de différence en matière de survie globale et de récidives loco- régionales dans le traitement conservateur chez les femmes de plus et de moins 65 ans. Concernant le curage ganglionnaire, il a été noté par différents auteurs que l'indication du curage ganglionnaire diminuait avec l'âge avancé, comparé aux malades plus jeunes [30]. En effet, Pappo retrouve seulement 15,1\% de curages ganglionnaires faits chez les patientes de plus de 70 ans, contre 30,3\% chez les malades plus jeunes [31]. Pour cela, plusieurs raisons sont avancées, notamment l'augmentation de la morbidité post-opératoire ou encore la prescription plus importante d'hormonothérapie chez les femmes âgées, vu le taux élevé de récepteurs hormonaux positifs dans cette tranche d'âge.

Toute personne âgée sans comorbidité majeure doit bénéficier d'une irradiation complémentaire en fonction des indications habituelles, car le sous-traitement a un effet défavorable à long terme sur la survie. Plusieurs essais randomisés et une méta-analyse ont confirmé la réduction très significative des rechutes locales avec l'adjonction de la radiothérapie après chirurgie conservatrice [32-33-34]. Chez les patientes de 70 ans et plus, l'association chirurgie conservatrice et radiothérapie permet à 5 ans un contrôle local de l'ordre de $95 \%$. Dans une grande étude canadienne [35] ayant inclus 4836 femmes de 50 ans et plus (dont $50 \% \geq 65$ ans) traitées par chirurgie conservatrice $( \pm$ tamoxifène et/ou chimiothérapie), $11 \%$ n'ont pas eu de radiothérapie complémentaire. Ces patientes ont globalement un taux de récidives locales de $9 \%$ versus $3 \%$ pour celles ayant eu une radiothérapie, avec parallèlement une diminution de la survie de $94 \%$ à $91 \%$. Des résultats similaires sont observés après mastectomie. De ce fait, afin de faciliter l'irradiation des personnes âgées, en particulier en cas de comorbidités, des schémas de radiothérapie hypofractionnée peuvent être utilisés [36]. Une étude de l'institut Curie, incluant 367 patientes ( $\geq 70$ ans) traitées entre 1995 et 1999 a retrouvé, également avec un recul de 93 mois, des résultats comparables en termes de contrôle locorégional (93\% et $87 \%$ ) dans le groupe de traitement classique (317 cas) et dans celui ayant subi une irradiation hypofractionnée (50 cas). Aucune différence n'a été observée en termes de survie spécifique, de survie sans récidive et de survie sans métastases [37]. Dans notre études $77,14 \%$ des patientes avaient bénéficié d'une irradiation prophylactique et une seule patiente a eu un protocole hypofractionné. Aucune complication grave n'a été notée. 
Les femmes âgées sont moins susceptibles de recevoir une chimiothérapie, en raison de plusieurs facteurs l'existence de comorbidités, la forte positivité des récepteurs hormonaux et l'inquiétude concernant d'éventuelles toxicités. Son indication est peu documentée dans la littérature et reste marginale, la chimiothérapie est indiquée si le risque de rechute à 10 ans est supérieur ou égal à $5 \%$. La toxicité des produits de chimiothérapie est potentiellement plus importante après 70 ans en raison des modifications de la pharmacocinétique et de la pharmacodynamique des médicaments. La baisse de capacité de régénération tissulaire peut augmenter les effets secondaires des traitements. La diminution de l'intensité de dose qui peut être tentante afin de favoriser la tolérance est toujours délétère pour l'efficacité [7]. Dans notre série, La chimiothérapie a été prescrite en adjuvant chez 20 malades, en situation néoadjuvante chez 7 malades (20\%) et l'indication était purement palliative chez 2 patientes.

Les caractéristiques biologiques et évolutives des cancers du sein de la femme âgée amènent à considérer l'approche hormonale comme la plus logique tant en phase adjuvante qu'en phase métastatique. L'apport des inhibiteurs de l'aromatase (IA) chez la femme ménopausée a été largement prouvé et comparé à celui du Tamoxifène dans de nombreuses études, notamment par l'étude ATAC (l'Anastrozole prolongerait significativement la survie sans récidives et diminuerait les métastases à distance et les cancers du sein controlatéral) [38]. Il faut cependant tenir compte des antécédents médico-chirurgicaux de la patiente, ainsi que des effets secondaires de chaque molécule pour la prescription de l'hormonothérapie. Dans notre série 23 malades $(65,71 \%)$ ont été mises sous hormonothérapie en adjuvante alors que l'indication d'une hormonothérapie palliative chez 3 patientes, les antiaromatases ont été utilisés chez 18 malades. 5 patientes étaient sous tamoxifène.

Le pronostic des tumeurs du sein chez la femme âgée est favorable et la survie à 5 ans dépasse généralement $80 \%$. Elle reste légèrement inférieure à celle des patientes plus jeunes. Ceci serait dû à un diagnostic plus tardif des femmes âgées et surtout à des traitements moins intensifs ou incomplets en raison des co-morbidités. En effet, une analyse de Louwman [39] de la survie globale des malades âgées de plus de 70 ans en fonction des co-morbidités a démontré qu'en l'absence de co-morbidités, elle atteignait 93\%. En présence de deux co-morbidités ou plus, elle diminuait à $53 \%$.

\section{Conclusion:-}

Le cancer du sein de la femme âgée est de par sa fréquence croissante un problème major de santé. Malgré que le cancer du sein de la femme âgée présente un profil biologique plus favorable avec des récepteurs hormonaux positifs et une faible expression de l'HER2 et du Ki67 son pronostic globalement plus péjoratif s'explique principalement par un délai à la prise en charge et par un traitement souvent suboptimal, d'où la nécessité d'insister sur l'information des équipes soignantes et de la population sur l'intérêt de l'examen clinique annuel des seins chez la femme âgée et de prescrire une mammographie de dépistage tous les deux ans chez les patientes à risque.

Le facteur âge en lui-même ne doit pas être considéré comme un élément discriminant de l'offre de soins chez la femme âgée atteinte de cancer du sein, d'où la nécessité de standardiser les protocoles thérapeutiques, les échelles d'évaluation et de promouvoir la gériatrie au Maroc pour une bonne prise en charge de cette tranche d'âge.

\section{References:-}

1. Arpana M .Naik,M.D.,Kathy Joseph,M.D.,Marcia Harris,M.D.,Christine Davis,Richard Shapiro,M.D.,Karen L.Hiotis,M.D.Indigent breast cancer patients among all racial and ethnic groups present with more advanced disease compared with nationally reported data. The American Journal of Surgery 2003; 186: 400-403.

2. Haut Commissariat au Plan Prospective Maroc 2030;2006 ;p9-34

3. YANCIK R, WESLEY M-N, RIES L-A-G. Effect of age and comorbidity in postmenopausal breast cancer patients aged 55 years and older JAMA 2001;285:885-92

4. SATARIANO W-A, RAGLAND D-R. The effect of comorbidity on 3-year survival of women with primary breast cancer Ann Intern Med 1994;120(2):104-10

5. GIRONES R, TORREGROSA D, DIAZ-BEVERIDGE R. Comorbidity, disability and geriatric syndromes in elderly breast cancer survivors. Results of a single-center experience.

6. James BK, Whittmore AS, Paffenbarger RS. Combined effect of childbearing, mentrual events and body size on age-specific breast cancer risk. Am J Epidemiol 1988; 128: 962-979.Critical reviews of oncology/ hematology 2010;73:236-45

7. SERIN D, ESCOUTE M. Cancer du sein de la femme âgée Encycl Med chir, Gynécologie ; 689-A-20, 1999, 6 . 
8. Sasco AJ., Gendre I. Le cancer de la femme de plus de 70 ans. Facteurs de risques. In : cancer du sein de la femme de plus de 70 ans. XIX ème Journées de la SFSPM Avignon. Paris : Arnette 1997 : 35-49.

9. AQUINO J-P. Prise en charge des cancers mammaires et gynécologiques chez les personnes âgées. Ed Ecole Eur Oncol ;1998:23-7.

10. Martin LM, Le Pechoux C, Calitchi E et al. Management of breast cancer in the elderly. Eur J Cancer; 30A: 590-596.

11. HIEKEN T-J, NETTNIN S, VELASCO J-M. The vallue of sentinel lymph node biopsy in elderly breast cancer patients The American Journal of Surgery 2004; 188:440-2.

12. TRUONG P-T, LEE J, KADER H-A, SPEERS C-H, OLIVOTTO I-A. Locoregional recurrence risks in elderly breast cancer patients treated with mastectomy without adjuvant radiotherapy. European journal of cancer 2005;41 :1267-77.

13. CHAPGAR A-B, MCMASTERS K-M, MARTIN R-C, THOENE C, NURKO J-Y, EDWARDS M-J, et al Determinants of early distant metastatic disease in elderly patients with breast cancer The American journal of surgery 2006;192:317-21.

14. LEE J-C, TRUONG P-T, KADER H-A, SPEERS C-H, OLIVOTTO I-A. Postmastectomy radiotherapy reduces locoregional recurrence in elderly women with high risk breast cancer. Clinical oncology $2005 ; 17$ :623-9.

15. VELANOVICH V, GABEL M, WALKER E-M, DOYLE T-J, O'BRYAN R-M, SZYMANSKI W, et al. Causes for the undertreatment of elderly breast cancer patients: tailoring treatments to individual patientsJ Am Coll Surg 2002; 194 :8-13.

16. SERIN D, ESCOUTE M. Cancer du sein de la femme âgée Encycl Med chir,Gynécologie ; 689-A-20, 1999, 6p.

17. David P, Robert T, Herman R. The national cancer data base report on breast carcinoma characterestics and outcome in relation to age. Cancer $1996 ; 78: 1838-1843$.

18. Juhan V. Cancer du sein de la femme âgée de plus de 70 ans. Thèse Med. Marseille 1997.

19. BRAIN E.Cancer du sein après 70 ans en France Bulletin du cancer, vol 89-101-4

20. DURBECQ V, AMEYE L, VEYS I. A significant proportion of elderly patients develop hormone-dependant "luminal B" tumours associated with aggressive characteristics Critical reviews in oncology/hematology 2008; 67:80-92.J Am Coll Surg 2001; $192: 698-707$

21. MA C-D, ZHOU Q, NIE X-Q, LIU G-Y, DI G-H, WU J, et al. Breast cancer in Chinese elderly women: Pathological and clinical characteristics and factors influencing treatment patterns. Critical reviews of oncology/hematology 2009;71:258-65 Cancer 1996 ; $78: 1838-1843$.

22. Fisher CJ, Egan M, Smith P, wiks K, Millis R, Fentiman IS. Histopathology of breast cancer in relation to age. Br J Cancer 1997; 75 : 593-596

23. Jaquemier J. Anatomie pathologique et cancers du sein des femmes de plus de 70 ans. Dans: 19èmes journées nationales de la SFSPM. Paris: Arnette 1997 : 97-100.

24. DAIDONE M-G, CORADINI D, MARTELLI G, VENERONI S. Primary breast cancer in elderly women: biological profile and relation with clinical outcome Critical reviews in oncology/hematology 2003;45:313-25.

25. GENNARI R, CURIGLIANO G, ROTMENSZ N. Breast carcinoma in elderly women: features of disease presentation, choice of local and systemic treatments compared with younger postmenopasual patients. Cancer 2004;101:1302-10.

26. Lesur-Shwander A, Rios M. Epidemiologie descriptive du cancer du sein de la femme de plus de 70 ans. Dans: 19èmes journées nationales de la SFSPM. Paris: Arnette $1997: 23$ - 33.

27. SANDISON A-J, GOLD D-M, WRIGHT P. Breast conservation or mastectomy: treatment choice of women aged 70 years and older. Br J Surg. 1996;83:994-6.

28. Solin LJ, Schultz DJ, Fowble BL. Ten-year results of the treatment of early stage breast carcinoma in elderly women using breast conserving surgery and definitive breast irradiation. Int J Radiat Oncol Biol Phys 1995; 33: 45-51.

29. TRUONG P-T, BERNSTEIN V, WAI E, CHUA B, SPEERS C, OLIVOTTO I-A. Age-related variations in the use of axillary dissection: a survival analysi of 8038 women withT1-ST2 breast cancer Int J radiation oncology Biol. Phys 2002;54:794-803.

30. PAPPO I, KARNI T, SANDBANK J, DINUR I, SELLA A, STAHL-KENT V, et al. Breast cancer in the elderly: histological, hormonal and surgical characteristics the breast 2007;16:60-7.

31. Clarke M, Collins R, Darby S et al. Effects of radiotherapy and of differences in the extent of surgery for early breast cancer on local recurrence and 15-year survival: an overview of the randomized trials. Lancet 2005,366:2087-106

32. Cutuli B. La radiothérapie du cancer du sein chez le sujet âgé. Cancer Radiothérapie 2009;13: 615-22. 
33. Fourquet A, Cutuli B, Luporsi E et al. Standards, Options et Recommandations 2001 pour la radiothérapie des patientes atteintes d'un cancer du sein infiltrant non métastatique. Cancer Radiotherapy 2002;6:1-2

34. Truong P, Bernstein V, L esperance $M$ et al. Radiotherapy omission after breast-conservative surgery is associated with reduced breast cancer-specific survival in elderly women with breast cancer. Am J Surg 2006;1941:749-55.

35. COURDI A, ORTHOLAN C, HANNOUN-LEVI J-M, FERRERO J-M, LARGILLIER R, BALUMAESTRO $\mathrm{C}$, et al. Long-term results of hypofractionated radiotherapy and hormonal therapy without surgery for breast cancer in elderly patients. Radiotherapy and oncology $2006 ; 79: 156-61$.

36. Kirova YM, Campana F, Savignoni A et al. Breast conserving treatment in the elderly: long-term results of adjuvant hypofractionated and normofractionated radiotherapy. Int J Radiat Oncol Biol Phys 2009;75:76-81.

37. SERIN D, ESCOUTE M.Cancer du sein de la femme âgé Encycl Med chir, Gynécologie; 689-A-20, 1999, 6p.

38. PETRAKIS I, PARASKAKIS S. Breast cancer in the elderly Archives of gerontology and geriatrics $2010 ; 50$ :179-84.

39. LOUWMAN W-J, JANSSEN-HEIJNEN M-L, HOUTERMAN S. Less extensive treatment and inferior prognosis for breast cancer patient with comorbidity: a population-based study.Eur J Cancer 2005;41:779-85. 